\title{
P04-1-120
}

Poster session

\section{Influence of the maternal immunoactivation on the neuron-microglia interactions in young offspring}

\section{Agnieszka Basta-Kaim, Boguslawa Budziszewska, Katarzyna Kotarska, Katarzyna Chamera, Ewa Trojan, Natalia Bryniarska}

\section{Experimental Neuroendocrinology, Institute of Pharmacology PAS, Poland}

Background: Accumulating evidence suggests that microglial activation plays a key role in the pathogenesis of various brain disorders, including depression. Activated microglia produce a wide range of factors, whose prolonged or excessive release may be crucial for the brain pathogenesis. Recently a growing body of evidence draws attention to the neuron - glia crosstalk as a mechanism of resolution of microglia activation. Among others the role of fractalkine (CX3CL1) and its receptor (CX3CR1) as well as CD200 and CD200 receptor (CD200R) has been identified in these processes. The expression of ligands on neurons and appropriate receptors (CX3CR1 and CD200R) mainly on microglial cells establishes a unique communication system between these cells within the central nervous system.

We set out to asses, whether the maternal immune activation, based on the bacterial endotoxin (lipopolisaccharide, LPS) administration affects CX3CL1-CX3CR1 and CD200-CD200R communications in the brain of young offspring.

Methods: Pregnant rats were injected subcutaneously with LPS at a dose of $2 \mathrm{mg} / \mathrm{kg}$ every second day from the seventh day of pregnancy until the delivery. Control pregnant rats were left undisturbed in their homecages. At 7 days of age, control and prenatally LPS-treated rats were decapitaded and hippocampi and frontal cortices were dissected. Then the protein levels of CX3CL1, CX3CR1, CD200 and CD200R was measured by western blot or ELISA methods.

Results: The data showed that LPS treatment increased the CX3CL1 level in cortex, but not in hippocampus of young offspring. On the other hand in hippocampus and frontal cortex of young animals the diminished level of CD200R was observed.

Conclusion: Our results provide evidence that prenatal immune activation leads to significant changes in neuronmicroglia axis in young offspring. It may be postulated that the presence of these disturbances during the critical neurodevelopmental period may play important role in the occurrence of schizophrenia-like behavioral deficits, observed in adult animals in this neurodevelopmental model of schizophrenia.

The work was supported by Polish National Science Centre (NCN 2015/19/B/NZ7/02394) and partially by the Statutory Funds of the Institute of Pharmacology Polish Academy of Sciences. 